\section{Industry leaders cite barriers to sequencing in trials}

In September, the Norwalk, Connecticutbased Multiple Myeloma Research Foundation (MMRF) announced they will be partnering with Millennium, a wholly-owned Takeda subsidiary in Cambridge, Massachusetts, in an eight-year stratified medicine initiative to follow 1,000 individuals, characterize their genetic and molecular biomarkers, and track their molecular response to treatment. And more recently, Foundation Medicine of Cambridge,

Massachusetts, announced that it will use its nextgeneration exome sequencing test in collaboration with the M.D. Anderson Cancer Center in Houston, to help match patients to experimental drugs. Despite these early steps, however, many still question whether the time is ripe to move towards broadly incorporating next- generation sequencing into the clinical trials

A panel convened on September 26 in Providence, Rhode Island, as part of Cambridge Healthtech Institute's "NGX: Applying Next-Generation Sequencing" conference cautioned that sequencing is unlikely to transform clinical trials in the near term. Even so, Bradley Smith, vice president of Drug Development Partnerships at contract research organization (CRO) Quintiles Transnational of Durham, North Carolina, does feel that NGS could accelerate trial planning by helping to match patients with the right treatment. "What we're working toward now... is a situation where we'll have pools of patients that are pre-screened," he said. "We could find a patient [who doesn't respond to] one drug but is positive for something else, and then switch them over to another."

Smith's enthusiasm was tempered by lya Khalil, executive vice president and cofounder of GNS Healthcare, who emphasized that genome sequence alone may be insufficient and that trial planners should also use next-generation sequencing to obtain transcriptomic data describing mRNA and microRNA levels alongside more conventional protein and metabolic biomarkers. Data analysis at this scale poses a logistical challenge, and her company is developing computational strategies to render these disparate data-points directly usable.

Indeed, many companies working at the clinical front lines remain hesitant to incorporate next-generation sequencing-driven biomarker hunting into their standard workflow. The complexities of turning such data into therapeutically meaningful indicators may be part of the problem, but Smith suggested that many are simply wary of increasing trial costs and the risk of being stuck with obsolete equipment as technology evolves. Panelists also pointed out that the use of genome-derived biomarkers remains a regulatory void, without clear guidance from the US Food and Drug Administration (FDA). "The technology is moving just as fast as it can," said Richard Resnick, CEO of software company GenomeQuest. "The FDA in some ways is just trying to keep up." Questions also remain about how to make genomic data available to clinicians and trial planners in a secure manner that assuages patients' privacy concerns.

Equally importantly, the reliability of current next-generation sequencing platforms may be insufficient to confidently validate biomarkers. Toby Bloom, director of bioinformatics for the genome sequencing facility at the Broad Institute in Cambridge, Massachusetts, pointed out that every next-generation sequencing platform introduces some sort of bias, necessitating the collection of larger quantities of data to bolster accuracy. "We're not quite there yet," said Bloom. Khalil concurred that the capacity to consistently obtain accurate and readily comparable sequence data across clinical trial phases will be essential.

Nevertheless, panelists saw clinical and academic laboratories poised to drive progress if industry fails to take the lead. Smith cited the MMRF's recently launched CoMMpass initiative (http://www.themmrf.org/research-programs/commpass-study/), as a positive example. "That's going to be an incredible amount of data, and that foundation is focused on enhancing drug development," said Smith.

Khalil concluded that it may take a big 'success story' to get industry excited about incorporating next-generation sequencing into drug development. She described promising beginnings in her own company's ongoing efforts with oncology partners to use genomic profiling to improve stratification in cancer trials, with an eye toward selecting the right patients from the start. "We know that there's a more rational way to do this," said Khalil. Michael Eisenstein

\section{IN brief}

\section{$\mathrm{NIH}$ tightens conflict rules}

In a long anticipated move, the US National Institutes of Health tightened its disclosure requirements for funding applicants. The original rules established in 1995 called for disclosure when a researcher-or his/her spouse or children - received at least $\$ 10,000$ in payments or $5 \%$ equity from companies or outside institutions. The new rules issued in August reduce that amount to $\$ 5,000$ or any equity at all in a private company. Some are disappointed, however, that one rumored change was not included: the requirement for universities to post potential conflicts in publicly accessible web sites. Universities criticized the proposal. "That's a disappointment, because I do think it would be useful to have that information publicly available. I do think that awareness is higher and there will probably be more activity around disclosures," says Lisa Bero, professor of clinical pharmacy and health policy at the University of California San Francisco, Tom Stossel, professor at Harvard Medical School, is a critic of the new policy. He says that no one has produced evidence that financial entanglements are harmful, and too much disclosure could lead to a witch hunt that undermines collaborations between academia and industry. "The old regulations didn't require very much. The new ones are going to open a Pandora's Box."

Jim Kling

\section{Bispecific biologics rush}

In late August, Vancouver-based Zymeworks inked a deal worth up to $\$ 187$ million plus royalties to advance bi-specific antibodies for Merck of Whitehouse Station, New Jersey. A week later Vienna-based f-star announced a collaboration with Merck Serono of Geneva to discover bi-specific IgGs against the pharma's targets, in a deal worth up to $\$ 676$ million plus royalties. Such major deals in rapid succession suggest that bi-specific antibody platforms have matured enough to attract investment from big pharma. "Deals tend to come in lumps," says Carl Gordon, a partner at healthcare investment firm OrbiMed Advisors based in New York. Gordon points to a May publication (J. Clin. Oncol. 29, 2386-2390, 2011) showing strong efficacy for Carlsbad, California-based Micromet's blinatumomab in a phase 2 trial for acute lymphocytic leukemia. Micromet's antibody is a bi-specific T-cell engager (BiTE), which binds CD19 on B cells and a CD3 site for T cells. "That is probably a proof of concept for the whole field," Gordon says. Zymeworks' platform consists of in silicodesigned heterodimeric IgG scaffolds containing two different but complementary heavy chain subunits. f-star's bi-specific antibodies can be engineered with three antigen binding sites, so that they are heterovalent for one epitope and monovalent for another. The technology has matured enough to solve some inherent problems, and to generate "data that are interesting enough to get those deals," adds Jean-François Formela, partner at Atlas Venture in Cambridge, Massachusetts, and a member of f-star's board of directors. Josh P Roberts 